\section{AB0053 THE ROLE OF ANGIOPOIETIN-LIKE PROTEINS TYPES 3 AND 4 IN THE DEVELOPMENT OF RENAL DYSFUNCTION IN PATIENTS WITH RHEUMATOID ARTHRITIS}

V. Aleksandrov ${ }^{1,2}$, L. Shilova ${ }^{1}$, A. Aleksandrov ${ }^{1,2},{ }^{1}$ Volgograd State Medical University, Department of Hospital Therapy, Volgograd, Russian Federation ${ }^{2}$ Federal State Budgetary Institution «Research Institute of Clinical and Experimental Rheumatology named after A.B. Zborovsky», Department of Clinical and Laboratory Diagnostics, Volgograd, Russian Federation

Background: Rheumatoid arthritis (RA) often contributes to the development of kidney disease. Angiopoietin-like proteins can be target markers for studying cardiorenal complications of RA [1].

Objectives: Assessment of the correlation of serum concentrations of angiopoietin-like proteins types 3 and 4 (ANGPTL 3 and 4 ) with the progression of renal dysfunction in RA patients.

Methods: 114 patients with reliable RA (90.4\% of women, $9.6 \%$ of men) aged 21 to 80 years (mean age $55.4 \pm 11.2$ years old), disease duration $-11.18 \pm 9.03$ years, positive for rheumatoid factor (RF-IgM) - $63.2 \%$, positive for anti-citrullinated protein antibody (ACPA) - 59.7\%) were examined. The laboratory examination included the determination of serum concentrations of angiopoietin-like protein type 3 (Human Angiopoietin-like Protein 3 ELISA, Bio Vendor, Czech Republic) and type 4 (RayBio Human ANGPTL4 ELISA Kit; RayBiotech, USA). To assess renal function in RA patients we used the calculated glomerular filtration rate (GFR) according to the CKD-EPI formula [2], taking into account the height and weight of a particular patient without indexing by body surface area. GFR values $<60 \mathrm{ml} / \mathrm{min} / 1.73 \mathrm{~m} 2$ were regarded as a certain decrease, and GFR values from 60 to $89 \mathrm{ml} / \mathrm{min} / 1.73 \mathrm{~m} 2$ - as a slight decrease in global kidney function. Results: The concentration of ANGPTL3 in the blood serum of RA patients $(\mathrm{n}=$ 158) was $641.9 \pm 224.5 \mathrm{ng} / \mathrm{ml}$, and that of ANGPTL4 $(\mathrm{n}=158)-3.15$ [0.77; 12.1] $\mathrm{ng} / \mathrm{ml} .74 .7 \%(\mathrm{n}=118)$ were considered positive for the presence of ANGPTL3; $49.4 \%(n=78)$ of RA patients were recognized as positive for the presence of ANGPTL4. The average glomerular filtration rate in RA patients was $74.0 \pm$ $18.6 \mathrm{ml} / \mathrm{min}$. More than a half of the examined RA patients had GFR ranging from 89 to $60 \mathrm{ml} / \mathrm{min} / 1.73 \mathrm{~m} 2$ (C1 - 21.5\%; C2 - 58.9\%; C3 - 19.6\%). No sharp reduce of renal function (GFR $<30 \mathrm{ml} / \mathrm{min} / 1.73 \mathrm{~m} 2$ ) corresponding to CKD C4-5 stages was recorded. Negative correlations of average strength were found between GFR and the level of ANGPTL $3(r=-0.32, p<0.001)$ and ANGPTL 4 ( $r S=-0.31, p<0.001)$, as well as with age $(r S=-0.28, p<0.001)$, the duration of $\mathrm{RA}(r S=-0.22, p=0.005)$ and blood pressure increase $(r S=-0.25, p=0.001)$. On the basis of GFR measurements, patients were divided into three groups: group I - optimal renal function ( $>90 \mathrm{ml} / \mathrm{min}$ ); group II - a slight decrease in renal function $(89-60 \mathrm{ml} / \mathrm{min})$; group III - reduced renal function $(<59 \mathrm{ml} / \mathrm{min})$.

Table 1. Content of ANGPTL 3 and 4 in RA patients with different GFR, $\mathrm{ng} / \mathrm{ml}$

\begin{tabular}{llll}
\hline & Group I $(\mathrm{n}=34)$ & Group II $(\mathrm{n}=93)$ & Group III $(\mathrm{n}=31)$ \\
\hline ANGPTL 3 & $533,4 \pm 161,7^{|-||| \mid}$ & $650,0 \pm 223,9$ & $733,2 \pm 244,1$ \\
ANGPTL 4 & $0,77[0,28 ; 3,6]^{1-|-|,|-| I I}$ & $3,3[0,93 ; 12,1]$ & $6,48[1,52 ; 19,3]$ \\
\hline
\end{tabular}

Note: upper case indicates intergroup differences at $p<0.05$.

There was a significant difference in the content of ANGPTL3 in patients of the first group and the patients of the third group $(\mathrm{H}$-test $=6.55, \mathrm{p}=0.032$ ) and ANGPTL4 in the group of patients with normal renal function (group I) and groups of RA patients with decreased GFR (group I- II: H-test $=10.7, p=0.001$; groups I-III: H-test $=20.1, p<0.001)$. ANGPTL4 indices also had intergroup differences (groups II-III: H-test $=7.2, p=0.007$ ) with GFR less than $90 \mathrm{ml} / \mathrm{min}$.

Conclusion: Chronic rheumatoid inflammation potentiates the development of renal dysfunction according to our data in $78.5 \%$ of patients. It is also accompanied by an increase in the content of ANGPTL types 3 and 4 in the blood of RA patients. A better understanding of the actions and mechanisms of ANGPTL may be of paramount importance for the development of effective ways of treatment for cardiorenal complications in RA.

REFERENCES:

[1] Aleksandrov A., Aleksandrov V., Shilova L. Study of the role of angiopoietin-like protein type 4 in metabolic disorders caused by inflammation in rheumatoid arthritis. Ann Rheum Dis. 2020;79(s1):1341. doi: 10.1136/ annrheumdis-2020-eular.4558.

[2] KDIGO 2012 clinical practice guideline for the evaluation and management of chronic kidney disease. Kidney Int Suppl. 2013:3:1-150.

Disclosure of Interests: None declared

DOI: 10.1136/annrheumdis-2021-eular.2275

$\mathrm{AB} 0054$

\section{IS TEA CONSUMPTION ASSOCIATED WITH RISK OF} RHEUMATOID ARTHRITIS?

H. Westerlind ${ }^{1}$, I. Palmqvist ${ }^{1}$, S. Saevarsdottir ${ }^{1,2}$, L. Alfredsson ${ }^{3}$, L. Klareskog ${ }^{1}$,

D. DI Giuseppe ${ }^{1} .{ }^{1}$ Karolinska Institute, Department of Medicine Solna,
Stockholm, Sweden; ${ }^{2}$ School of Health Sciences, University of Iceland, Faculty of Medicine, Reykjavik, Iceland; ${ }^{3}$ Karolinska Institute, Institute of Environmental Medicine, Stockholm, Sweden

Background: Only few studies have looked at the association between tea consumption and risk of rheumatoid arthritis (RA) with inconclusive results.

Objectives: To estimate the association between tea consumption and risk of RA in a large population-based case-control study.

Methods: We used data from the Swedish Epidemiological Investigation of RA (EIRA), a population-based case-control study including incident RA cases with 2 controls randomly matched from the general population, based on age, sex and residential area at the date of diagnosis. All participants filled in a comprehensive questionnaire on lifestyle factors, including a 124-item food frequency questionnaire (FFQ). Data from October 2005 - May 2018 was used

Odds ratios (ORs) with 95\% confidence intervals (Cls) were estimated using conditional logistic regression, overall and stratified by anti-citrullinated protein antibody (ACPA) and smoking status. We adjusted for smoking status, coffee and alcoho consumption, educational level and BMI. The dose-response trend of the association between tea consumption and risk of rheumatoid arthritis was estimated using restricted cubic spline with knots at $0,0.29$, and 2.29 cups per day. Missing tea values $(44.8 \%)$ were imputed based on the zero-consumption assumption. A sensitivity analysis was performed to evaluate the influence of the assumption on the main result, with $70 \%$ of the missing randomly imputed as no consumption, and the remaining $30 \%$ randomly assigned to the other categories (10\% per category). Results: We included 2237 cases and 4661 controls. Controls were more likely to drink $\geq 2$ tea cups/day compared to RA cases (22.1\% vs. $19.7 \%$ ).

The crude odds of developing RA was $22 \%$ lower (OR=0.78, 95\% Cl: 0.66-0.92) among those who consumed $\geq 2$ tea cups/day compared to those who drank $<1$ cup/day (irregular drinkers). After adjustment for covariates, the inverse association was less pronounced and only borderline significant $(\mathrm{OR}=0.85,95 \% \mathrm{Cl}$ : $0.71-1.01)$. A similar OR was observed among non-tea consumer ( $O R=0.82,95 \%$ Cl: 0.70-0.95). The odds of developing ACPA positive RA (but not ACPA negative) was statistically significant lower among participants drinking $>=2$ cups/day compared to irregular tea drinkers (OR=0.76, $95 \% \mathrm{Cl}: 0.62-0.94)$. In analyses stratified by smoking, an inverse association between tea intake and RA was found only among current smokers (OR=0.58, 95\% Cl: $0.37-0.92)$, while no association was found in never smoker. The sensitivity analysis showed results similar to the main analysis, although the OR in the no consumption category was not significant $(\mathrm{OR}=0.88,95 \% \mathrm{Cl}: 0.76-1.02)$ while the $\mathrm{OR}$ in the highest category was statistically significant $(\mathrm{OR}=0.85,95 \% \mathrm{Cl}: 0.73-0.99)$.

\begin{tabular}{|c|c|c|c|c|}
\hline & \multicolumn{4}{|c|}{ Overall tea consumption } \\
\hline & 0 cups/day & $<1$ cup/day & $1-2$ cups/day & $>=2$ cups/day \\
\hline \multicolumn{5}{|l|}{ Overall } \\
\hline Number & $955 / 1941$ & $453 / 847$ & $388 / 845$ & $441 / 1028$ \\
\hline OR crude* & $0.89(0.77-1.03)$ & Ref & $0.84(0.71-1.00)$ & $0.78(0.66-0.92)$ \\
\hline OR adjusted ${ }^{ \pm}$ & $0.82(0.70-0.95)$ & Ref & $0.87(0.73-1.04)$ & $0.85(0.71-1.01)$ \\
\hline \multicolumn{5}{|l|}{ ACPA positive } \\
\hline Number & $637 / 1156$ & $321 / 527$ & $268 / 501$ & $277 / 630$ \\
\hline OR adjusted & $0.81(0.67-0.97)$ & Ref & $0.88(0.71-1.09)$ & $0.76(0.62-0.94)$ \\
\hline \multicolumn{5}{|l|}{ ACPA negative } \\
\hline Number & $312 / 571$ & $129 / 221$ & $119 / 251$ & $162 / 288$ \\
\hline OR adjusted & $0.87(0.66-1.14)$ & Ref & $0.87(0.63-1.19)$ & $1.07(0.78-1.46)$ \\
\hline \multicolumn{5}{|l|}{ Never smokers } \\
\hline Number & $290 / 859$ & $164 / 436$ & $142 / 433$ & $213 / 563$ \\
\hline OR adjusted & $0.90(0.72-1.14)$ & Ref & $0.88(0.68-1.15)$ & $1.04(0.81-1.33)$ \\
\hline \multicolumn{5}{|c|}{ Current smokers } \\
\hline Number & $296 / 371$ & $89 / 96$ & $59 / 78$ & $59 / 99$ \\
\hline OR adjusted & $0.81(0.57-1.14)$ & Ref & $0.83(0.52-1.31)$ & $0.58(0.37-0.92)$ \\
\hline
\end{tabular}

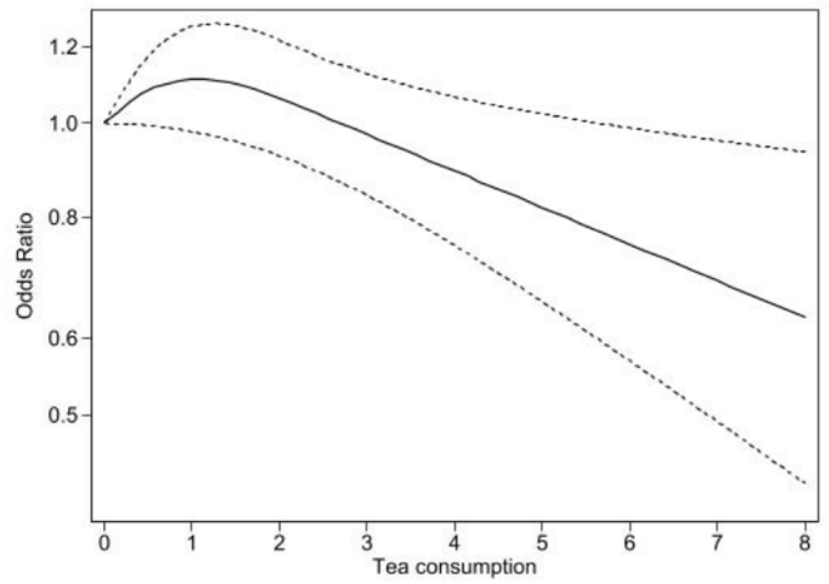

Figure 1. Dose-response odds ratio for risk of rheumatoid arthritis (RA) by tea consumption. 